\title{
SPECTRAL CONVERGENCE OF MULTIQUADRIC INTERPOLATION
}

\author{
by MARTIN BUHMANN and NIRA DYN
}

(Received 26th July 1991)

In this paper, we consider interpolants on $h \cdot \mathbb{Z}^{n}$ from the closure of the space spanned by translates of the function $\left(\|\cdot\|^{2}+1\right)^{\beta / 2}\left(\beta>-n\right.$ and not an even nonnegative integer) along $h \cdot \mathbb{Z}^{n}$. We show that these interpolants approximate a function, whose Fourier transform satisfies certain asymptotic conditions, up to an error of order $h^{p}$, on any compact domain in $\mathbb{R}^{n}$, where $p$ is only restricted by the smoothness of the function.

1991 Mathematics subject classification: 41A05, 41A25, 41A30, 41A63.

\section{Introduction}

This paper is concerned with the degree of approximation over a compact domain, achieved by interpolation on $h \cdot \mathbb{Z}^{n}$ from

$$
V_{h}=\text { closure } \operatorname{span}\left\{\phi(\|\cdot-j h\|): j \in \mathbb{Z}^{n}\right\},
$$

where $\|\cdot\|$ denotes the Euclidean norm, and where the radial function $\phi(\|\cdot\|)$ is of multiquadric type

$$
\phi(r)=\left(r^{2}+1\right)^{\beta / 2}, \quad \beta>-n, \quad \frac{1}{2} \beta \notin \mathbb{Z}_{+} .
$$

Here the closure is taken relative to the topology of uniform convergence over compact domains in $n$-dimensional real space.

It is shown in Buhmann [4, Sections 3-4], that there exists a unique interpolant to the data $\left\{f(j h): j \in \mathbb{Z}^{n}\right\}$ of the form

$$
J_{h} f(x)=\sum_{j \in \mathbb{Z}^{n}} f(j h) \chi\left(h^{-1} x-j\right), \quad x \in \mathbb{R}^{n}
$$

where the "cardinal function" $\chi \in V_{1}$ satisfies $\chi(j)=\delta_{j 0}, j \in \mathbb{Z}^{n}$, and $|\chi(x)|$ decays algebraically (i.e. as a certain negative power of $\|x\|$ ) at infinity. Moreover, it is known that

$$
\left\|J_{h} f-f\right\|_{\infty}=O\left(h^{\beta+n}\right), \quad h \rightarrow 0,
$$

for $f \in C^{\lceil\beta\rceil+n}\left(\mathbb{R}^{n}\right)$ with bounded derivatives of order $\lceil\beta\rceil+n-2,\lceil\beta\rceil+n-1$ and $\lceil\beta\rceil+n$, 
where $\lceil\beta\rceil$ denotes the least integer $\geqq \beta$; see Buhmann [2, Example 5.2] and also [4] for the cases when $n+\beta$ is not an odd integer and Powell [13] for the case when $n+\beta$ is an odd integer. Strictly speaking Powell provides a proof of (1.4), when $n+\beta$ is an odd integer, only for $\beta=1$, but his proof generalizes easily. In fact, unless $n+\beta$ is an odd integer, the boundedness requirement on the $([\beta\rceil+n-2)$-th derivative can be waived $[2,4]$. If $n+\beta$ is an even integer, the boundedness requirement on the $(\lceil\beta\rceil+n-1)$-th derivative can be dropped too [2-4]. It is clear from (1.2) and the fact $\chi \in V_{1}$ that

$$
\chi\left(h^{-1} \cdot\right) \in \text { closure } \operatorname{span}\left\{\phi_{h}(\|\cdot-j h\|): j \in \mathbb{Z}^{n}\right\},
$$

with

$$
\phi_{h}(r)=\left(r^{2}+h^{2}\right)^{\beta / 2} .
$$

Thus the radial function whose translates along $h \cdot \mathbb{Z}^{n}$ are used, changes with $h$.

In this paper we study the convergence rates in case the radial basis function (1.2) is fixed for all grids $h \cdot \mathbb{Z}^{n}, h>0$, and we obtain much stronger results.

It follows from the extension of the Strang and Fix conditions to the case of approximating operators of the form

$$
Q_{h} f(x)=\sum_{j \in \mathbb{Z}^{n}} f(j h) \psi\left(h^{-1} x-j\right), \quad x \in \mathbb{R}^{n},
$$

with $\psi$ an algebraically decaying function (see, e.g., Light and Cheney [10]), that

$$
\left\|f-Q_{h} f\right\|_{\infty}=O\left(h^{l+\alpha}\right), \quad h \rightarrow 0,
$$

can only be obtained with positive $\alpha$ for a suitable class of differentiable functions $f$ if $\pi_{l} \subset$ closure span $\left\{\psi(\cdot-j): j \in \mathbb{Z}^{n}\right\}$, where $\pi_{l}$ is the space of polynomials over $\mathbb{R}^{n}$ of total degree $\leqq l$. In fact, result (1.4) is obtainable by first noting that

$$
J_{h} p=p, \quad \forall p \in \pi_{\lceil\beta\rceil+n-1},
$$

and we cannot obtain (1.4) with $O\left(h^{\beta+n}\right)$ replaced by $O\left(h^{\beta+n^{+}+1}\right)$ since $\pi_{\Gamma \beta 7+n} \not V_{h}$. For further details, the reader is referred to Dyn [6] and Powell [13].

The results for the interpolation operators

$$
I_{h} f(x)=\sum_{j \in Z^{n}} f(j h) \chi_{h}(x-j h), \quad x \in \mathbb{R}^{n},
$$

where the cardinal function $\chi_{h} \in V_{h}$ satisfies $\chi_{h}(j h)=\delta_{j 0}, j \in \mathbb{Z}^{n},\left(\chi_{1}\right.$ being the same as $\left.\chi\right)$ are of a different nature, due to the fact the $\chi_{h}$ is not a scaled version of $\chi_{1}$ and that the spaces $\left\{V_{h}\right\}_{h>0}$ are obtained from translating the same radial basis function (1.2). In particular $V_{l h} \subset V_{h}, l \in \mathbb{Z}$. Now let $f: \mathbb{R}^{n} \rightarrow \mathbb{R}$ be a function which can be modified by subtracting a polynomial term so as to have a distributional Fourier transform $\hat{f}_{*}(t)$ that decays, for a fixed $v>2 n+\beta$, as $\|t\|^{-v}$ for large $\|t\|$, and satisfies for some $\delta>0$ 


$$
\int_{0<\|t\|<\delta}\left|\hat{f}_{*}(t)\right|\|t\|^{n+\beta} d t<\infty .
$$

It will be shown that we have then, for any $p<v-n$,

$$
\left\|f-I_{h} f\right\|_{\infty, \Omega}=\sup _{x \in \Omega}\left|f(x)-I_{h} f(x)\right|=O\left(h^{p}\right), \quad h \rightarrow 0 .
$$

Here $\Omega$ is any compact domain in $\mathbb{R}^{n}$. We call this spectral convergence since the convergence rate is essentially linked to the decay of $f$ 's Fourier transform at infinity and hence to $f$ 's smoothness. Of course we may conclude that the error estimate above is true for all positive $p$ as soon as $\hat{f}_{*}(t)$ decays faster than any integral power of $\|t\|$ at infinity. This convergence of $I_{h} f$ to $f$ is partly based on the important property of the spaces $V_{h}$ that local approximations to all monomials can be constructed in $V_{h}$, with error of one order higher than the degree of the monomial.

Related results on approximation orders by quasi-interpolation on $h \cdot \mathbb{Z}^{n}$, based on translates along $h \cdot \mathbb{Z}^{n}$ of the exponential box-spline $B_{h} \neq B_{1}\left(h^{-1} \cdot\right)$ are obtained by Dyn and Ron [7], using local approximations to monomials by the underlying space of exponential-polynomials.

A first observation of arbitrary orders of convergence in the context of radial function approximation is due to Madych and Nelson [11,12], who show that scattered data interpolation with any of the radial functions $\phi$ in (1.2) converges as $O\left(h^{p}\right)$, for all $p \in \mathbb{Z}_{+}$, on a bounded domain $\Omega$, as the data points become dense in a domain containing $\Omega$. This result applies to functions in the reproducing kernel Hilbert space induced by $\phi$, which is a space of very smooth functions with exponentially decaying Fourier transforms. Wu and Schaback [15] also prove this result using a different approach.

Throughout this paper we use standard multi-index notation. For $x, y \in \mathbb{R}^{n},\|x\|^{2}=$ $\sum_{k=1}^{n} x_{k}^{2},\langle x, y\rangle=\sum_{k=1}^{n} x_{k} \cdot y_{k}, x^{y}=\prod_{k=1}^{n} x_{k}^{y_{k}}$, and for $\alpha \in \mathbb{Z}_{+}^{n}, \alpha !=\prod_{k=1}^{n} \alpha_{k} !,|\alpha|=\sum_{k=1}^{n} \alpha_{k}$, $D_{x}^{\alpha}=\prod_{k=1}^{n}\left(\partial^{\alpha_{k}} / \partial x_{k}^{a_{k}}\right)$. We will also often write $r$ instead of $\|x\|$ as the argument of radially symmetric functions, i.e. $\phi(r)$ instead of $\phi(\|x\|), \hat{\phi}(r)$ instead of $\hat{\phi}(\|x\|)$ etc.

\section{Main results}

We need two preliminary results. The first one is a consequence of results in Buhmann $[4, \S 3]$ relating to the existence of cardinal functions of interpolation with radial basis functions. For its statement, recall that the distributional Fourier transform of $\phi_{y}(r)=\left(r^{2}+\gamma^{2}\right)^{\beta / 2}, \beta>-n$ and $\frac{1}{2} \beta \notin \mathbb{Z}_{+}$, is

$$
\hat{\phi}_{\gamma}(r)=\frac{2 \pi^{n / 2}}{\Gamma\left(-\frac{1}{2} \beta\right)} K_{(n+\beta) / 2}(\gamma r)\left(\frac{r}{2 \gamma}\right)^{-(n+\beta) / 2}, r>0
$$

Jones [9, p. 532]. Here, $K_{v}$ denotes a modified Bessel function $[1$, p. 374]. We also introduce the constant 


$$
\omega:= \begin{cases}2 & \text { if } \frac{1}{2}(n+\beta) \in \mathbb{Z}_{+}, \\ 1 & \text { otherwise, }\end{cases}
$$

for use in the statement of this theorem and thereafter.

Theorem 1. Let $\phi_{\gamma}(r)=\left(r^{2}+\gamma^{2}\right)^{\beta / 2}$ for $\beta>-n$ and $\frac{1}{2} \beta \notin \mathbb{Z}_{+}$. Then the coefficients

$$
c_{k}=\frac{1}{(2 \pi)^{n}} \int_{[-\pi, \pi]^{n}} \frac{e^{i\langle k, t\rangle}}{\sum_{l \in \mathbb{Z}^{n}} \hat{\phi}_{\gamma}(\|t-2 \pi l\|)} d t, \quad k \in \mathbb{Z}^{n},
$$

provide a cardinal function

$$
\chi(x)=\sum_{k \in Z^{n}} c_{k} \phi_{y}(\|x-k\|), \quad x \in \mathbb{R}^{n}
$$

satisfying $\chi(j)=\delta_{0 j}, j \in \mathbb{Z}^{n}$, with a Fourier transform

$$
\hat{\chi}(t)=\frac{\hat{\phi}_{\gamma}(\|t\|)}{\sum_{l \in Z^{n}} \hat{\phi}_{\gamma}(\|t-2 \pi l\|)}, \quad t \in \mathbb{R}^{n} .
$$

Moreover, for $\|x\| \rightarrow \infty$,

$$
|\chi(x)| \leqq A_{0}(1+\|x\|)^{-n-\omega(n+\beta)}
$$

with $A_{0}$ depending on $\gamma$ but independent of $x$.

For the statement of the next theorem, we introduce the notation

$$
\sigma_{h}(t):=\frac{1}{\sum_{l \in \mathbb{Z}^{n}} \hat{\phi}\left(\left\|t-h^{-1} 2 \pi l\right\|\right)}, \quad t \in \mathbb{R}^{n} .
$$

Theorem 2. Let $\phi(r)=\left(r^{2}+1\right)^{\beta / 2}$ for $\beta>-n$ and $\frac{1}{2} \beta \notin \mathbb{Z}_{+}$. Then, for every positive $h$, the coefficients

$$
c_{k}^{h}=\frac{h^{n}}{(2 \pi)^{n}} \int_{[-\pi, \pi]^{n}} e^{i\langle k, t\rangle} \sigma_{h}\left(h^{-1} t\right) d t, \quad k \in \mathbb{Z}^{n},
$$

provide a cardinal function

$$
\chi_{h}(x)=\sum_{k \in Z^{n}} c_{k}^{h} \phi(\|x-k h\|), \quad x \in \mathbb{R}^{n},
$$

satisfying $\chi_{h}(h j)=\delta_{j 0}, j \in \mathbb{Z}^{n}$, with a Fourier transform

$$
\hat{\chi}_{h}(t)=h^{n} \hat{\phi}(\|t\|) \sigma_{h}(t), \quad t \in \mathbb{R}^{n} .
$$

Moreover, for $\|x\| \rightarrow \infty$,

$$
\left|\chi_{h}(x)\right| \leqq A_{1}(1+\|x\|)^{-n-\omega(n+\beta)}
$$

where $A_{1}$ depends on $h$ but not on $x$.

Proof. It is easy to check that the $\chi(x)$ of Theorem 1 with $\gamma=h^{-1}$ equals $\chi_{h}(x h)$. This yields the theorem. 
We can now state the main result of this paper.

Theorem 3. Let $\phi(r)=\left(r^{2}+1\right)^{\beta / 2}$ for $\beta>-n$ and $\frac{1}{2} \beta \notin \mathbb{Z}_{+}$, and let $\Omega \subset \mathbb{R}^{n}$ be a compact domain. Suppose that $f: \mathbb{R}^{n} \rightarrow \mathbb{R}$ satisfies $|f(x)|=O\left((1+\|x\|)^{\omega(n+\beta)-\varepsilon}\right)$ for a positive $\varepsilon$. Suppose that there is a polynomial $P \in \pi_{[\omega(n+\beta)]-1}$ such that the distributional Fourier transform $\hat{f}_{*}$ of $f-P$ is in $C\left(\mathbb{R}^{n} \backslash\{0\}\right)$ and satisfies (1.9) for some $\delta>0$ and $\left|\hat{f}_{*}(t)\right| \leqq$ $A_{2}\|t\|^{-v}$ for a fixed $v>2 n+\beta$ and $\|t\| \geqq \delta$, where $A_{2}$ is a positive constant which does not depend on $t$. Then the interpolant (1.8) gives for any $p<v-n$ and for small $h$

$$
\left\|f-I_{h} f\right\|_{\infty, \Omega} \leqq A_{3} h^{p}
$$

where $A_{3}$ does not depend on $h$.

Before proving the theorem, we make several observations. Firstly, we note that (1.8) is well-defined for every positive spacing $h$ and for every $f$ that satisfies the bound given in the statement of the theorem, because of the bound (2.2) on $\chi_{h}$. We also note that the conditions on $f$ 's distributional Fourier transform imply that $f$ is p-times continuously differentiable for $p<v-n$. The assumptions on $f$ 's Fourier transform we make do not imply that $f$ belongs to the Hilbert space studied in Madych and Nelson, and $\mathrm{Wu}$ and Schaback, because there it is required that $P=0$ and

$$
\int_{\mathbf{R}^{n}} \hat{\phi}(\|t\|)^{-1}\left|\hat{f}_{*}(t)\right|^{2} d t<\infty
$$

for which our conditions on $\hat{f}_{*}$ both at the origin and at infinity are insufficient.

We finally point out that if $f$ is a polynomial of degree less than $n+\beta$, then the error estimate in the theorem is trivially true, because such polynomials are reproduced by the interpolation process (this follows from the analysis in Buhmann $[4, \S 5]$ ). If, however, $\omega=2$ and $f$ is a polynomial of degree at least $n+\beta$ and less than $2(n+\beta)$, then the error estimate is nontrivial. Powell [13] showed an error estimate of this type in the univariate case when $\beta=1$ and $f$ is a quadratic polynomial. In fact, his estimate is stronger, in that it shows exponential convergence of the interpolant to the function, whereas our estimate only gives convergence of any integral order.

The proof of Theorem 3 occupies the rest of this section. We first give an outline of the proof. We remark that, unless stated otherwise, all the constants $A_{i}, i=4,5, \ldots$, which occur in the sequel are positive and independent of $h$ and $x$ as long as $x$ is confined to the compact set $\Omega$ and $h$ is positive and, say, less than 1 . Dependencies of $A_{i}$ on other variables that are relevant to the analysis will be noted as arguments of $A_{i}$.

The central idea of the proof is to construct for any given positive integer $p$ and $z \in h \cdot \mathbb{Z}^{n}$ a "local approximation" $G_{z}: \mathbb{R}^{n} \rightarrow \mathbb{R}$ to $f$ such that

$$
\left|f(x)-G_{z}(x)\right| \leqq A_{4} h^{p}, \quad \forall x, h:\|x-z\|<h<A_{5},
$$

$A_{4}$ and $A_{5}$ being not only independent of $x$ and $h$, but also of $z$. We require $G_{z}$ to be of the form 


$$
G_{z}(x)=\sum_{j \in N} \mu_{j, z}^{h} \phi(\|x-z-j h\|), \quad x \in \mathbb{R}^{n}
$$

with $N \subset \mathbb{Z}^{n}$ a finite subset which does not depend on $z$ and $h$. We then prove the identity

$$
I_{h}\left[\phi\left(\left\|\cdot-z^{\prime}\right\|\right)\right](x)=\phi\left(\left\|x-z^{\prime}\right\|\right), \quad x \in \mathbb{R}^{n}, \forall h>0,
$$

whenever $z^{\prime} \in h \cdot \mathbb{Z}^{n}$, and we use (2.6) to show that, for any fixed $x$ and for $z \in h \cdot \mathbb{Z}^{n}$ such that $\|x-z\|<h<A_{5}$,

$$
\begin{aligned}
\left|f(x)-I_{h} f(x)\right| & \leqq\left|f(x)-G_{z}(x)\right|+\left|G_{z}(x)-I_{h} f(x)\right|=\left|f(x)-G_{z}(x)\right|+\left|I_{h}\left[G_{z}-f\right](x)\right| \\
& \leqq A_{4} h^{p}+\left|I_{h}\left[G_{z}-f\right](x)\right| .
\end{aligned}
$$

Finally, we show that the second term in (2.7) can be bounded above by a multiple of $h^{p}$, the multiplier being the same for all small enough $h$ and for all $x \in \Omega$. The estimate (2.3) follows.

In order to find a function (2.5) such that (2.4) is true, we will make use of the following proposition:

Proposition 4. Let $\phi(r)=\left(r^{2}+1\right)^{\beta / 2}$ for $\beta>-n$ and $\frac{1}{2} \beta \notin \mathbb{Z}_{+}$. Then, for every given positive $d$ and every $\alpha \in \mathbb{Z}_{+}^{n}$, there is a function

$$
H_{\alpha}^{d}(x)=\sum_{j \in N_{\alpha}} v_{j}^{\alpha, d} \phi(\|x-j d\|), \quad x \in \mathbb{R}^{n}
$$

with $N_{\alpha} \subset \mathbb{Z}^{n}$ a finite subset which is independent of $d$, such that

$$
\left|x^{\alpha}-H_{\alpha}^{d}(x)\right| \leqq A_{6}(d, \alpha)\|x\|^{|\alpha|+1}, \quad \forall x:\|x\|<A_{7}(d, \alpha),
$$

and $H_{\alpha}^{d}$ can be chosen such that $\min _{d \in K} A_{7}(d, \alpha)>0$ and $\max _{d \in K} A_{6}(d, \alpha)<\infty$ if $K$ is $a$ compact interval in $\mathbb{R}_{>0}$.

When employing this result later on in order to form $G_{z}$ as a linear combination of the functions $H_{\alpha}^{d}$, we shall make a suitable choice of $d$ such that it is always an integral multiple of $h$ (so in particular $H_{\alpha}^{d} \in V_{h}$ as required), and such that it remains confined to a compact interval in $\mathbb{R}_{>0}$ when $h \rightarrow 0$.

Proof. If we take $A_{7}(d, \alpha)$ positive and small enough, we can expand (2.8) for any finite subset $N_{\alpha} \subset \mathbb{Z}^{n}$ and for all $\|x\|<A_{7}(d, \alpha)$ as follows:

$$
H_{\alpha}^{d}(x)=\sum_{j \in N_{\alpha}} v_{j}^{\alpha, d}\left(1+\|j d\|^{2}\right)^{\beta / 2} \sum_{k=0}^{\infty}\left(\begin{array}{c}
\beta / 2 \\
k
\end{array}\right)\left\{\frac{\|x\|^{2}-2\langle x, j d\rangle}{1+\|j d\|^{2}}\right\}^{k}
$$




$$
=\sum_{k=0}^{\infty}\left(\begin{array}{c}
\beta / 2 \\
k
\end{array}\right) \sum_{j \in N_{\alpha}} \eta_{j}^{\alpha, d}\left\{\frac{\|x\|^{2}-2\langle x, j d\rangle}{1+\|j d\|^{2}}\right\}^{k}
$$

with $\eta_{j}^{\alpha, d}=v_{j}^{\alpha, d}\left(1+\|j d\|^{2}\right)^{\beta / 2}$. Clearly, $A_{7}(d, \alpha)$ can be chosen to depend continuously on $d$. Hence $\min _{d \in K} A_{7}(d, \alpha)>0$ if $K$ is a compact interval. Furthermore, there is a constant $A_{8}(d, \alpha)$ such that, for $\|x\|<A_{7}(d, \alpha)$,

$$
\begin{gathered}
\left|H_{\alpha}^{d}(x)-\sum_{k=0}^{|\alpha|}\left(\begin{array}{c}
\beta / 2 \\
k
\end{array}\right) \sum_{j \in N_{\alpha}} \eta_{j}^{\alpha, d}\left\{\frac{\|x\|^{2}-2\langle x, j d\rangle}{1+\|j d\|^{2}}\right\}^{k}\right| \\
\leqq A_{8}(d, \alpha)\|x\|^{|\alpha|+1}
\end{gathered}
$$

In order that (2.8) satisfies (2.9), we now require a set of conditions on $N_{\alpha} \subset \mathbb{Z}^{n}$ and on the $\left\{\eta_{j}^{\alpha, d}\right\}_{j \in N_{\alpha}}$ which ensure that

$$
\begin{gathered}
\left|x^{\alpha}-\sum_{k=0}^{|\alpha|}\left(\begin{array}{c}
\beta / 2 \\
k
\end{array}\right) \sum_{j \in N_{\alpha}} \eta_{j}^{\alpha, d}\left\{\frac{\|x\|^{2}-2\langle x, j d\rangle}{1+\|j d\|^{2}}\right\}^{k}\right| \leqq A_{9}(d, \alpha)\|x\|^{|\alpha|+1}, \\
\forall x:\|x\|<A_{7}(d, \alpha),
\end{gathered}
$$

thus admitting $A_{6}(d, \alpha):=A_{8}(d, \alpha)+A_{9}(d, \alpha)$. Specifically, it suffices to require

$$
\left\{\begin{array}{c}
\sum_{j \in N_{\alpha}} \eta_{j}^{\alpha, d}(j d)^{\gamma}\left(1+\|j d\|^{2}\right)^{-k}=0, \quad \forall \gamma, k: 2 k-|\alpha| \leqq|\gamma| \leqq k<|\alpha|, \\
(-2)^{|\alpha|}\left(\begin{array}{c}
\beta / 2 \\
|\alpha|
\end{array}\right)\left(\begin{array}{c}
|\alpha| \\
\alpha
\end{array}\right) \sum_{j \in N_{\alpha}} \eta_{j}^{\alpha, d}(j d)^{\gamma}\left(1+\|j d\|^{2}\right)^{-|\alpha|}=\delta_{\alpha \gamma}, \quad \forall \gamma:|\gamma|=|\alpha|,
\end{array}\right.
$$

where $\left(\begin{array}{c}|\alpha| \\ \alpha\end{array}\right)$ is a multinomial coefficient. We have to demonstrate that there exist a finite $N_{\alpha} \subset \mathbb{Z}^{n}$ and coefficients $\left\{\eta_{j}^{\alpha, d}\right\}_{j \in N_{\alpha}}$ such that (2.12) holds. To this end, we note that we can rewrite conditions (2.12) as

$$
\left\{\begin{array}{cc}
\sum_{j \in N_{a}} \varrho_{j}^{\alpha, d}(j d)^{\gamma}\left(1+\|j d\|^{2}\right)^{|\alpha|-k}=0, & \forall \gamma, k: 2 k-|\alpha| \leqq|\gamma| \leqq k<|\alpha|, \\
(-2)^{|\alpha|}\left(\begin{array}{c}
\beta / 2 \\
|\alpha|
\end{array}\right)\left(\begin{array}{c}
|\alpha| \\
\alpha
\end{array}\right) \sum_{j \in N_{\alpha}} \varrho_{j}^{\alpha, d}(j d)^{\gamma}=\delta_{\alpha \gamma,} & \forall \gamma:|\gamma|=|\alpha|,
\end{array}\right.
$$


$\varrho_{j}^{\alpha, d}$ being $\eta_{j}^{\alpha, d}\left(1+\|j d\|^{2}\right)^{-|\alpha|}$. We can now show that there is a finite set $N_{\alpha} \subset \mathbb{Z}^{n}$ and a solution $\left\{\varrho_{j}^{\alpha, d}\right\}_{j \in N_{\alpha}}$ to (2.13) by proving that there is a set $N_{\alpha} \subset \mathbb{Z}^{n}$ such that the vector $\left\{(j d)^{\alpha}\right\}_{j \in N_{\alpha}}$ is not in the span of the vectors $\left\{\left\{(j d)^{\gamma}\left(1+\|j d\|^{2}\right)^{|\alpha|-k}\right\}_{j \in N_{a}}: 2 k-|\alpha| \leqq|\gamma| \leqq k<\right.$ $|\alpha|\}$ and $\left\{\left\{(j d)^{\gamma}\right\}_{j \in N_{a}}:|\gamma|=|\alpha|\right.$ but $\left.\gamma \neq \alpha\right\}$. This is equivalent to the claim that there is a finite set $N_{\alpha} \subset \mathbb{Z}^{n}$ such that, for any sets of coefficients $\left\{a_{\gamma}\right\}$ and $\left\{b_{\gamma}\right\}$, the polynomial

$$
x \mapsto x^{\alpha}+\sum_{\substack{|\gamma|=|\alpha| \\ \gamma \neq \alpha}} a_{\gamma} x^{\gamma}+\sum_{2 k-|\alpha| \leqq|\gamma| \leqq k<|\alpha|} b_{\gamma} x^{\gamma}\left(1+\|x\|^{2}\right)^{|\alpha|-k}
$$

is not identically zero on $d \cdot N_{\alpha}$. First of all, if (2.14) were identically zero on $\mathbb{P}^{n}$ for some sets of coefficients $\left\{a_{\gamma}\right\}$ and $\left\{b_{\gamma}\right\}$, then

$$
x^{\alpha}+\sum_{\substack{|y|=|\alpha| \\ y \neq \alpha}} a_{\gamma} x^{y}=-\sum_{2 k-|\alpha| \leqq|\gamma| \leqq k<|\alpha|} b_{y} x^{\gamma}\left(1+\|x\|^{2}\right)^{|\alpha|-k}, \quad x \in \mathbb{R}^{n}
$$

but since $|\alpha|-k \geqq 1$ on the right-hand side of (2.15), we may conclude from (2.15) that $1+\|x\|^{2}$ divides the homogeneous polynomial on the left-hand side of (2.15), a contradiction. Now, if we choose $N_{\alpha} \subset \mathbb{Z}^{n}$ to be a tensor product of $2|\alpha|+1$ points in each of the $n$ coordinate directions, then (2.14), which is a non-trivial polynomial of coordinate degree less than $2|\alpha|+1$, cannot vanish on $d \cdot N_{\alpha}$ for any set of coefficients. Thus there exists a solution $\left\{\eta_{j}^{\alpha, d}\right\}_{j \in N_{\alpha}}$ to (2.12), and this solution yields (2.11). Inequalities (2.10) and (2.11) imply (2.9). In order to show that $H_{\alpha}^{d}$ can be chosen such that $\max _{d \in K} A_{6}(d, \alpha)$ is bounded if $K$ is a compact interval, we note that $A_{6}(d, \alpha)$ depends continuously on the coefficients of $H_{\alpha}^{d}$ and we now show that these coefficients can be chosen to remain bounded if $d$ is confined to $K$. For each $d$ the coefficients of $H_{\alpha}^{d}$ solve an underdetermined linear system of equations whose coefficients depend continuously on $d$. Let $m$ be the rank of this system for a given $d_{0} \in K$. We can select a nonsingular $m \times m$ minor which remains nonsingular if $d$ is within a neighbourhood $U_{d_{0}}$ of $d_{0}$. Fixing the remaining $\left|N_{\alpha}\right|-m$ variables, we conclude that the solution of the underdetermined system depends continuously on $d$ as long as $d$ is confined to $U_{d_{0}}$. Now, if $K \subset \mathbb{R}_{>0}$ is compact, the covering $\bigcup_{d \in K} U_{d} \supset K$ has a finite number of sets $U_{d_{1}}, U_{d_{2}}, \ldots, U_{d_{k}}$ such that $\bigcup_{i=1}^{k} U_{d_{i}} \supset K$. Because the above selected solution to the underdetermined system depends continuously on $d$ for all $d$ in some $U_{d_{i}}$ and because there are finitely many $U_{d_{i}}$ that cover $K$, the solution vectors can be chosen to remain bounded if $d$ ranges over $K$, and so does $A_{6}(d, \alpha)$.

Now let

$$
A_{5}:=\min \left[\frac{1}{2}, \min _{\substack{|\alpha|<p \\ 1 / 2 \leqq d \leqq 3 / 2}} A_{7}(d, \alpha)\right]
$$


which is positive by Proposition 4. Further, for every positive $h$, let us choose $\kappa_{h} \in \mathbb{Z}_{+}$ such that $\left|h^{-1}-\kappa_{h}\right|<1$. Then we let $d:=h \kappa_{h}$ in Proposition 4. Thus, for $0<h<A_{5}$, the quantity $d$ is confined to the compact interval $\left[1-A_{5}, 1+A_{5}\right]$, which is a subset of $\left[\frac{1}{2}, \frac{3}{2}\right]$. Note in particular that $d \cdot \mathbb{Z}^{n} \subset h \cdot \mathbb{Z}^{n}$ by virtue of the choice of $d$. We now complete the proof of the existence of $(2.5)$ such that $(2.4)$ is true by letting

$$
G_{z}(x)=\sum_{|\alpha|<p} \delta_{\alpha, z}^{d} H_{\alpha}^{d}(x-z), \quad x \in \mathbb{R}^{n}
$$

where the quantities $d$ and $z$ are as defined above and where we define recursively

$$
\delta_{\alpha, z}^{d}=\left.\frac{1}{\alpha !} D_{x}^{\alpha}\left\{f(x)-\sum_{|\gamma|<|\alpha|} \delta_{y, z}^{d} H_{y}^{d}(x-z)\right\}\right|_{x=z} .
$$

We prove (2.4) by induction on $p$ as follows. Just for the following argument we will denote the function (2.16) by $G_{z}^{[p]}$, to indicate its dependence on $p$. Because

$$
\delta_{0, z}^{d}=f(z)
$$

it follows from Taylor's Theorem and Proposition 4 that the claim holds for $p=1$, i.e. that

$$
\left|G_{z}^{[1]}(x)-f(x)\right| \leqq A_{4}(1) h, \quad \forall x, h:\|x-z\|<h<A_{5},
$$

where here and in the following argument we write $A_{4}(p)$ instead of $A_{4}$, again in order to take notice of its dependence on $p$. Now suppose that we have

$$
\left|G_{z}^{[p]}(x)-f(x)\right| \leqq A_{4}(p) h^{p}, \quad \forall x, h:\|x-z\|<h<A_{5}
$$

Then, by the smoothness of both $f$ and $G_{z}^{[p]}$ and by definition (2.17),

$$
\left|f(x)-G_{z}^{[p]}(x)-\sum_{|\alpha|=p} \delta_{\alpha, z}^{d}(x-z)^{\alpha}\right| \leqq A_{10}(p) h^{p+1}, \quad \forall x, h:\|x-z\|<h<A_{5}
$$

Expression (2.19) implies

$$
\begin{aligned}
\left|G_{z}^{[p+1]}(x)-f(x)\right| & \leqq\left|\sum_{|\alpha|=p} \delta_{\alpha, z}^{d}\left\{H_{\alpha}^{d}(x-z)-(x-z)^{\alpha}\right\}\right|+\left|G_{z}^{[p]}(x)+\sum_{|\alpha|=p} \delta_{\alpha, z}^{d}(x-z)^{\alpha}-f(x)\right| \\
& \leqq \sup _{\substack{z \in B_{1}(\Omega) \\
1 / 2 \leqq d \leqq 3 / 2}}\left\{\sum_{|\alpha|=p}\left|\delta_{\alpha, z}^{d}\right| A_{6}(d, \alpha)\right\} h^{p+1}+A_{10}(p) h^{p+1}
\end{aligned}
$$


for all $\|x-z\|<h<A_{5}, B_{1}(\Omega)$ being the ball about the origin containing $\Omega$, whose surface has at least Euclidean distance 1 from $\Omega$. Estimate (2.20) admits the definition

$$
A_{4}(p+1)=\sup _{\substack{z \in B_{1}(\Omega) \\ 1 / 2 \leqq d \leqq 3 / 2}}\left\{\sum_{|\alpha|=p}\left|\delta_{\alpha, z}^{d}\right| A_{6}(d, \alpha)\right\}+A_{10}(p),
$$

and this proves (2.4).

Remark 5. In case $\left\|D^{\alpha} f\right\|_{\infty}$ is bounded for $|\alpha| \leqq p$, it follows from (2.17) that the bounds on $\left\{\left|\delta_{\alpha, z}^{d}\right|:|\alpha| \leqq p\right\}$ are independent of $z$, and so is $A_{10}(p)$ in (2.19). Thus (2.4) holds for all $x \in \mathbb{R}^{n}$ with appropriate $z \in h \cdot \mathbb{Z}^{n}$, and not only for $x \in \Omega$, the constant $A_{4}$ being independent of $x$ and $z$ for $x \in \mathbb{R}^{n}, z \in h \cdot \mathbb{Z}^{n}$, with $\|x-z\|<h<A_{5}$.

Next, we establish (2.6), namely the reproduction of the radial functions spanning $V_{h}$ by the interpolation operator $I_{h}$.

Proposition 6. Let $\phi(r)=\left(r^{2}+1\right)^{\beta / 2}$ for $\beta>-n$ and $\frac{1}{2} \beta \notin \mathbb{Z}_{+}$. Then, for every positive $h$ and every $k \in \mathbb{Z}^{n}$,

$$
I_{h}[\phi(\|\cdot-k h\|)](x)=\phi(\|x-k h\|), \quad x \in \mathbb{R}^{n} .
$$

Proof. Without loss of generality, we take $k=0$. Since $I_{h}[\phi(\|\cdot\|)]$ is a tempered distribution, we may first prove the claim in the sense of tempered distributions. Now, let $S_{m}$ be the set of rapidly decreasing test functions $s \in C^{\infty}\left(\mathbb{R}^{n}\right)$ which satisfy $D^{\gamma} \hat{s}(0)=0$ for all $|\gamma|<m$. We have for $m>\beta$ and $s \in S_{m}$

$$
\begin{aligned}
\int_{\mathbb{R}^{n}} I_{h}[\phi(\|\cdot\|)](x) s(x) d x & =\int_{\mathbb{R}^{n}} \sum_{j \in \mathbb{Z}^{n}} \phi(\|j h\|) \chi_{h}(x-j h) s(x) d x \\
& =\sum_{j \in \mathbb{Z}^{n}} \phi(\|j h\|) \int_{\mathbf{R}^{n}} \chi_{h}(x-j h) s(x) d x,
\end{aligned}
$$

where the interchange of summation and integration that leads to (2.22) will be justified later.

We now apply the Poisson Summation Formula [14, p. 251] to the sum (2.22) to obtain that it is the same as

$$
\begin{aligned}
\frac{1}{(2 \pi h)^{n}} \sum_{j \in \mathbf{Z}^{n}} \int_{\mathbf{R}^{n}} \hat{\phi}\left(\left\|y-h^{-1} 2 \pi j\right\|\right) \hat{\chi}_{h}(y) \hat{s}(-y) d y & =\frac{1}{(2 \pi h)^{n}} \int_{\mathbf{R}^{n}} \sum_{j \in \mathbf{Z}^{n}} \hat{\phi}\left(\left\|y-h^{-1} 2 \pi j\right\|\right) \hat{\chi}_{h}(y) \hat{s}(-y) d y \\
& =\frac{1}{(2 \pi)^{n}} \int_{\mathbf{R}^{n}} \eta(y) d y=\int_{\mathbf{R}^{n}} \phi(\|x\|) s(x) d x
\end{aligned}
$$

where $\eta(y):=\hat{\phi}(\|y\|) \hat{s}(-y)$. Here, the penultimate equality follows from (2.1) and the last 
equality follows from Parseval's Formula [9, Theorem 7.13, p. 224]. This proves the claim modulo a polynomial $q \in \pi_{M}$. However, since the asserted equality is true for all $x \in h \cdot \mathbb{Z}^{n}$ by the interpolation property, this polynomial has to vanish. We note that the application of the Poisson Summation Formula, and the interchanges in summation and integration that preceded and that followed it, are justified if we show that

and

$$
\sum_{j \in Z^{n}}|\phi(\|j h\|)| \int_{\mathbf{R}^{n}}\left|\chi_{h}(x-j h) s(x)\right| d x<\infty
$$

$$
\sum_{j \in \mathbf{Z}^{n}} \int_{\mathbf{R}^{n}}\left|\hat{\chi}_{h}\left(y-h^{-1} 2 \pi j\right) \eta(y)\right| d y<\infty
$$

In fact, both estimates follow from Lemma 4 in Buhmann and Dyn [5], but we prove them here explicitly for the convenience of the reader.

We establish the first inequality: In order to do this, we will have to introduce constants $A_{11}, A_{12}, A_{13}$ which may depend on $h$ (but not on $x$ or $j$ ), but we shall take no notice of this, because it is irrelevant to our proof. Using (2.2), we have

$$
\int_{\mathbf{R}^{n}}\left|\chi_{h}(x-j h) s(x)\right| d x \leqq A_{1} \int_{\mathbf{R}^{n}}(1+\|x-j h\|)^{-n-\omega(n+\beta)}|s(x)| d x .
$$

Moreover, mimicking an argument Light and Cheney [10] use in their proof of Lemma 4.14, we have, whenever $\|x\| \leqq \frac{1}{2}\|j h\|$,

$$
(1+\|x-j h\|)^{-n-\omega(n+\beta)} \leqq(1+\|j h\|-\|x\|)^{-n-\omega(n+\beta)} \leqq\left(1+\frac{1}{2}\|j h\|\right)^{-n-\omega(n+\beta)} .
$$

Conversely, whenever $\|x\| \geqq \frac{1}{2}\|j h\|$, then of course $|s(x)| \leqq A_{11}\left(1+\frac{1}{2}\|j h\|\right)^{-n-\omega(n+\beta)}$. Therefore

$$
(1+\|x-j h\|)^{-n-\omega(n+\beta)}|s(x)| \leqq A_{12}\left(1+\frac{1}{2}\|j h\|\right)^{-n-\omega(n+\beta)}\left\{(1+\|x-j h\|)^{-n-\omega(n+\beta)}+|s(x)|\right\} .
$$

The three estimates (2.24)-(2.26) imply that the right-hand side of (2.24) is at most

$$
\begin{aligned}
& A_{1} A_{12}\left(1+\frac{1}{2}\|j h\|\right)^{-n-\omega(n+\beta)} \int_{\mathbf{R}^{n}}\left\{(1+\|x-j h\|)^{-n-\omega(n+\beta)}+|s(x)|\right\} d x \\
& =A_{1} A_{12}\left(1+\frac{1}{2}\|j h\|\right)^{-n-\omega(n+\beta)}\left\{\int_{\mathbf{R}^{n}}(1+\|y\|)^{-n-\omega(n+\beta)} d y+\int_{\mathbf{R}^{n}}|s(x)| d x\right\}
\end{aligned}
$$

This expression is, in turn, at most $A_{13}\left(1+\frac{1}{2}\|j h\|\right)^{-n-\omega(n+\beta)}$, which proves the first claim.

The second series can be seen to converge absolutely by noting that the argument of the last paragraph can be applied again, to show that the terms in the sum decay exponentially, due to the exponential decay of both $\hat{\chi}_{h}$ and $\eta$ that follows from the exponential decay of the modified Bessel functions $[1$, p. 378]. 
Remark 7. Proposition 6 holds in a more general setting of radial functions. Specifically, it is possible to show that it is true for Buhmann's class of "admissible" radial basis functions [4], including, among many others, $\phi(r)=r^{\beta}$, with $\beta>0, \frac{1}{2} \beta \notin \mathbb{Z}_{+}$, and $\phi(r)=r^{2 k} \log r, k=1,2, \ldots$

Finally, we have to prove that the second term on the right-hand side of $(2.7)$ can be bounded above by a constant multiple of $h^{p}$ if $\|x-z\|<h<A_{\mathbf{s}}$.

To this end we observe that

$$
\begin{aligned}
& \quad I_{h}\left[f-G_{z}\right](x)=\sum_{j \in \mathbf{Z}^{\eta^{n}}}\left[f(j h)-G_{z}(j h)\right] \chi_{h}(x-j h) \\
= & \lim _{\varepsilon \rightarrow 0^{+}} \sum_{j \in \mathbf{Z}^{\mathbf{Z}^{n}}}\left[f_{\varepsilon}(j h)-G_{z, \varepsilon}(j h)\right] \chi_{h}(x-j h),
\end{aligned}
$$

where $f_{\varepsilon}(x):=e^{-\varepsilon\|x\| \|^{2}} f(x)$ and $G_{z, \varepsilon}(x):=e^{-\varepsilon\|x\|^{2}} G_{z}(x)$. Expressions (2.28) and (2.27) are the same because the limit in $(2.28)$ can be taken inside the series by absolute convergence of the series in (2.27) and (2.28). We apply the Poisson Summation Formula to (2.28) and obtain that it is the same as

$$
\lim _{\varepsilon \rightarrow 0^{+}} \frac{1}{(2 \pi h)^{n}} \sum_{j \in \mathbf{Z}^{n}} \int_{\mathbf{R}^{n}} e^{i\left\langle x, t-h^{-1} 2 \pi k\right\rangle} \hat{\chi}_{h}\left(t-h^{-1} 2 \pi k\right)\left[\hat{f}_{\varepsilon}(t)-\hat{G}_{0, \varepsilon}(t)\right] d t,
$$

where we have now chosen $z=0$ without loss of generality. The application of the Poisson Summation Formula is justified because the argument we have used in the penultimate paragraph of the proof of Proposition 6 (or indeed Lemma 4 in [5]) can be used to show that for all positive $\varepsilon$

$$
\int_{\mathbf{R}^{n}}\left|\hat{\chi}_{h}\left(t-h^{-1} 2 \pi k\right)\right|\left|\hat{f}_{\varepsilon}(t)-\hat{G}_{0, \varepsilon}(t)\right| d t \leqq A_{14}(h, \varepsilon)(1+\|k\|)^{-v} .
$$

The first fact we have to employ in order to apply that argument is the exponential decay of $\hat{\chi}_{h}$. Secondly, we use the rapid (i.e. faster than any negative power) decay of $\hat{G}_{0, e}$ which can be established by standard arguments of Fourier analysis, due to the infinite differentiability of $G_{0, e}$ and the integrability of its derivatives. Finally, we use the estimate $\left|\hat{f}_{\varepsilon}(t)\right| \leqq A_{15}(\varepsilon)(1+\| t|t|)^{-v}$, which is also due to the differentiability of $f_{\varepsilon}$ and the integrability of its derivatives.

We now observe that (2.29) is the same as the following expression.

$$
\begin{aligned}
\lim _{\varepsilon \rightarrow 0^{+}} \frac{1}{(2 \pi h)^{n}} \int_{\mathbb{R}^{n}}\left\{e^{i\langle x, t\rangle} \hat{\chi}_{h}(t)\left[\hat{f}_{\varepsilon}(t)-\hat{G}_{0, \varepsilon}(t)\right]\right. \\
\\
\left.\quad+\sum_{k \in Z^{n} \backslash\{0\}} e^{i\left\langle x, t-h^{-1} 2 \pi k\right\rangle} \hat{\chi}_{h}\left(t-h^{-1} 2 \pi k\right)\left[\hat{f}_{\varepsilon}(t)-\hat{G}_{0, \varepsilon}(t)\right]\right\} d t
\end{aligned}
$$




$$
=\lim _{\varepsilon \rightarrow 0^{+}} \frac{1}{(2 \pi)^{n}} \int_{\mathbf{R}^{n}} e^{i\langle x, t\rangle}\left[\hat{f}_{\varepsilon}(t)-\hat{G}_{0, \varepsilon}(t)\right] d t
$$

$+\lim _{\varepsilon \rightarrow 0^{+}} \frac{1}{(2 \pi h)^{n}} \int_{\mathbf{R}^{n}} \sum_{k \in \mathbf{Z}^{n}\{\langle 0\}} e^{i\langle x, t\rangle}\left(e^{-2 \pi i h^{-1}\langle x, k\rangle}-1\right) \hat{\chi}_{h}\left(t-h^{-1} 2 \pi k\right)\left[\hat{f}_{\varepsilon}(t)-\hat{G}_{0, \varepsilon}(t)\right] d t$.

Expression (2.30) is in modulus at most $A_{4} h^{p}$ because it is identical to $\lim _{\varepsilon \rightarrow 0^{+}}\left[f_{\varepsilon}(x)-G_{0, \varepsilon}(x)\right]=\left[f(x)-G_{0}(x)\right]$ and because of (2.4). Our next goal is to estimate expression (2.31). To this end, recall definition (2.1) which relates $\hat{\chi}_{h}\left(t-h^{-1} 2 \pi k\right)$ to $\hat{\phi}\left(\left\|t-h^{-1} 2 \pi k\right\|\right)$ and $\sigma_{h}(t)$. For every nonzero $k$, there is no singularity at $t=0$ in $\hat{\phi}\left(\left\|t-h^{-1} 2 \pi k\right\|\right)$. Hence, the facts that $\sigma_{h}$ has a $\left.(n+\Gamma \beta\rceil\right)$-th order zero at the origin and is $C^{n+[\beta]}$ in a neighbourhood of zero if $\omega=1$ (this follows from the asymptotic behaviour of the modified Bessel function at the origin, see $[1, p .375])$ and $C^{2 n+2 \beta}$ in a neighbourhood of zero if $\omega=2$ (see [3, pp. 31f.] for the case $\beta=1$, which immediately generalises), and the assumption (1.9) imply that the following integral converges absolutely and is the same as (2.31):

$$
\begin{gathered}
\frac{1}{(2 \pi)^{n}} \int_{\mathbf{R}^{n}} \sum_{k \in \mathbb{Z}^{n} \backslash\langle 0\}} e^{i\langle x, t\rangle}\left(e^{-2 \pi i h^{-1}\langle x, k\rangle}-1\right) \hat{\phi}\left(\left\|t-h^{-1} 2 \pi k\right\|\right) \\
\times \sigma_{h}(t)\left[\hat{f}_{*}(t)+(2 \pi)^{n} P\left(i D_{t}\right) \delta(t)-\hat{G}_{0}(t)\right] d t .
\end{gathered}
$$

The modulus of (2.32) is less than

$$
\begin{aligned}
& \int_{\mathbf{R}^{n} \mid B_{1 / h}(0)} \sum_{k \in \mathbb{Z}^{n} \backslash\{0\}} \hat{\phi}\left(\left\|t-h^{-1} 2 \pi k\right\|\right) \sigma_{h}(t)\left|\hat{f}_{*}(t)-\hat{G}_{0}(t)\right| d t \\
& \quad+\int_{B_{1 / h}(0)} \sum_{k \in \mathbf{Z}^{n} \backslash\{0\}} \hat{\phi}\left(\left\|t-h^{-1} 2 \pi k\right\|\right) \sigma_{h}(t)\left|\hat{f}_{*}(t)-\hat{G}_{0}(t)\right| d t \\
& +2 P\left(i D_{t}\right)\left\{\sum_{k \in \mathbb{Z}^{n} \backslash\{0\}} e^{i\langle x, t\rangle}\left(e^{-2 \pi i h^{-1}\langle x, k\rangle}-1\right) \hat{\phi}\left(\left\|t-h^{-1} 2 \pi k\right\|\right) \sigma_{h}(t)\right\}_{t=0},
\end{aligned}
$$

where $B_{1 / h}(0)$ denotes the closed ball of radius $h^{-1}$ about the origin. Now assume that $h$ is at most $\delta^{-1}$, where $\delta$ is a constant from the statement of Theorem 3. Then the integral (2.33) is at most

$$
\begin{aligned}
\int_{\mathrm{R}^{n} \backslash B_{1 / h}(0)}\left|\hat{f}_{*}(t)-\hat{G}_{0}(t)\right| d t & \leqq \int_{\mathrm{R}^{n} \mid B_{1 / h(0)}}\left|\hat{f}_{*}(t)\right|+\left|\hat{G}_{0}(t)\right| d t \\
& \leqq-A_{16} \int_{h^{-1}}^{\infty} r^{n-v-1} d r+A_{17} h^{p} \\
& \leqq A_{18} h^{p}
\end{aligned}
$$


Here we have in particular used the exponential decay of $\hat{G}_{0}$ at infinity that follows from the bound $|\hat{\phi}(r)| \leqq A_{19} e^{-r}$ for large $r$, which is a consequence of the asymptotic exponential decay of the modified Bessel functions. Using this bound once again and using the bound $K_{v}(z)>2^{v-1} \Gamma(v) e^{-z} z^{-v}$ from Ismail [8], we see that (2.34) can be bounded above by

$$
A_{20} \int_{B_{1 / h}(0)} \frac{\sum_{k \in Z^{n} \mid\{0\}} e^{-h^{-1}\|t h-2 \pi k\|}}{e^{-\| t||}\|t\|^{-n-\beta}+\sum_{l \in Z^{n} \backslash\{0\}} e^{-h^{-1}\|t h-2 \pi l\|}\|t h-2 \pi l\|^{-n-\beta} h^{n+\beta}}\left|\hat{f}_{*}(t)-\hat{G}_{0}(t)\right| d t .
$$

This integral converges absolutely, for the same reasons which imply that (2.32) converges absolutely. It is at most a constant multiple of

$$
\begin{aligned}
& \max _{\|t\|<h^{-1}} \frac{\sum_{k \in Z^{n} \backslash\{0\}} e^{-h^{-1}\|t h-2 \pi k\|}\|t\|^{-n-\beta}}{e^{-\|t\|}\|t\|^{-n-\beta}+\sum_{l \in Z^{n} \backslash\{0\}} e^{-h^{-1}\|t h-2 \pi l\| !}\|t h-2 \pi l\|^{-n-\beta} h^{n+\beta}} \\
& \times \int_{\mathbf{R}^{n}}\|t\|^{n+\beta}\left|\hat{f}_{*}(t)-\hat{G}_{0}(t)\right| d t<A_{21} \max _{\|t\|<h^{-1}} e^{\|t\| \|} \sum_{k \in \mathbf{Z}^{n} \backslash\{0\}} e^{-h^{-1}\|t h-2 \pi k\|} .
\end{aligned}
$$

This expression can be bounded above by

$$
\begin{aligned}
A_{21} \max _{\|t\|<h^{-1}} e^{2\|t\|} \sum_{k \in \mathbb{Z}^{n} \backslash\{0\}} e^{-h^{-1}\|2 \pi k\|} & =A_{21} e^{2 h^{-1}} \sum_{k \in \mathbb{Z}^{n} \backslash\{0\}} e^{-h^{-1}\|2 \pi k\|} \\
& \leqq A_{21} e^{-4 h^{-1}} \sum_{k \in \mathcal{Z}^{n} \backslash\{0\}} e^{-0.2 h^{-1}\|k\|} \leqq A_{22} e^{-4 h^{-1}} .
\end{aligned}
$$

We finally address expression (2.35). As pointed out earlier, $\sigma_{h}$ has a zero of order $n+\lceil\beta\rceil$ at the origin, hence (2.35) vanishes if $\omega=1$ (since then $P$ 's degree has to be less than $n+\beta)$. Otherwise, we employ the fact that not only $|\hat{\phi}(r)| \leqq A_{19} e^{-r}$ for large $r$ but that the same asymptotic behaviour is observed by $\hat{\phi}$ 's derivatives, due to the asymptotic behaviour of derivatives of modified Bessel functions at infinity (as can be derived from the formulas for the derivatives of Bessel functions Abramowitz and Stegun give [1, p. 376]). Hence (2.35) can be bounded above by

$$
A_{23} \sum_{k \in \mathbf{Z}^{n} \backslash\{0\}} e^{-h^{-1}|| 2 \pi k \|} \leqq A_{24} e^{-4 h^{-1}}
$$

This shows that the theorem is true.

Corollary 8. Let $f: \mathbb{R}^{n} \rightarrow \mathbb{R}$ satisfy the assumptions of Theorem 3 and assume also that $\left\|D^{\alpha} f\right\|_{\infty}<\infty$ for $|\alpha| \leqq p$, where $p<v-n$. Then, for small enough $h$,

$$
\left\|f-I_{h} f\right\|_{\infty} \leqq A_{25} h^{p},
$$

where $A_{25}$ does not depend on $h$. 
Acknowledgement. It is a pleasure to thank M. J. D. Powell for his help with the research for this paper.

\section{REFERENCES}

1. M. Abramowitz and I. A. Stegun, Handbook of Mathematical Functions (Dover Publications, 1970).

2. M. D. BuhmanN, Multivariable Interpolation using Radial Basis Functions (Ph.D. Dissertation, University of Cambridge, 1989).

3. M. D. Buhmann, Multivariate interpolation in odd-dimensional Euclidean spaces using multiquadrics, Constr. Approx. 6 (1990), 21-34.

4. M. D. Buhmann, Multivariate cardinal interpolation with radial-basis functions, Constr. Approx. 6 (1990), 225-255.

5. M. D. Buhmann and N. Dyn, Error estimates for multiquadric interpolation, in Curves and Surfaces (P.-J. Laurent, A. LeMẻhauté, L. L. Schumaker, eds., Academic Press, New York, 1991), 51-58.

6. N. DYN, Interpolation and approximation by radial and related functions, in Approximation Theory VI (C. K. Chui, L. L. Schumaker, J. D. Ward, eds., Academic Press, 1989), 211-234.

7. N. DYN and A. RoN, Local approximation by certain spaces of exponential polynomials, approximation order of exponential box splines, and related interpolation problems, Trans. Amer. Math. Soc. 319 (1990), 381-403.

8. M. E. H. Ismall, Complete monotonicity of modified Bessel functions (ICM-Report 88-013, University of South Florida, 1988).

9. D. S. Jones, The Theory of Generalized Functions (Cambridge University Press, Cambridge, 1982).

10. W. A. Light and E. W. Cheney, Quasi-interpolation with translates of a function having non-compact support, Constr. Approx. 8 (1992), 35-48.

11. W. R. MADYch and S. A. Nelson, Error bounds for multiquadric interpolation, in Approximation Theory VI (C. K. Chui, L. L. Schumaker, J. D. Ward, eds., Academic Press, 1989), 413-416.

12. W. R. Madych and S. A. Nelson, Multivariate interpolation and conditionally positive definite functions II, Math. Comp. 54 (1990), 211-230.

13. M. J. D. PowelL, The theory of radial basis function approximation in 1990 , in Advances in Numerical Analysis II (W. A. Light, ed., Clarendon Press, Oxford, 1992), 105-210.

14. E. M. Stein and G. Weiss, Introduction to Fourier Analysis on Euclidean Spaces (Princeton University Press, Princeton, 1971).

15. Z. Wu and R. Schaback, Local error estimates for radial basis function interpolation of scattered data (Report University of Göttingen, 1990).

Department of Applied Mathematics

and Theoretical Physics

UNIVERSITY OF CAMBRIDGE

Cambridge CB3 9EW

ENGLAND
Raymond and Beverly Sackler Faculty of Exact SCIENCES

Tel Aviv University

Tel Aviv

ISRAEL 